\section{Plasmonic surface enhancement of dual fluorescence and phosphorescence emission from organic semiconductors: effect of exchange gap and spin-orbit coupling $\dagger$}

\author{
Debangshu Chaudhuri, ${ }^{* a b}$ Dongbo Li, ${ }^{a}$ Eva Sigmund, ${ }^{c}$ H. Wettach, ${ }^{c}$ Sigurd Höger ${ }^{c}$ and \\ John M. Lupton ${ }^{\text {ad }}$
}

Received 13th March 2012, Accepted 7th May 2012

DOI: $10.1039 / \mathbf{c} 2 \mathrm{cc} 31843 \mathrm{a}$

Dual singlet-triplet fluorescence-phosphorescence emitting compounds demonstrate that plasmonic surface enhancement is controlled solely by the underlying oscillator strength of a transition: metal-free compounds with weak spin-orbit coupling show no enhancement in phosphorescence efficiency even though fluorescence is amplified.

Excitement in the area of plasmonics stems from the ability to detect processes that are otherwise undetectable. Collective electron oscillations at metal surfaces experience strong spatial localization, which results in extremely intense and localized electromagnetic fields. This phenomenon is at the heart of all surface-enhanced spectroscopic processes. ${ }^{1}$ The intense local field can relax several selection rules, thereby allowing transitions that are otherwise forbidden, and are therefore inaccessible to regular spectroscopy. While observation of forbidden Raman modes in surface-enhanced Raman scattering (SERS) is well documented, ${ }^{2}$ the same is not as common for electronic transitions. Particularly noteworthy is a report on the observation of dipole-forbidden, but quadrupole-allowed transitions in conjugated oligoenes near silver films. ${ }^{3}$ A crucial question to explore is hence whether metal nanostructures can also enhance dipole-forbidden radiative recombination from triplet excited states, phosphorescence. The necessity arises because $75 \%$ of radical-pair recombination events in an organic light-emitting diode (OLED) lead to triplet excited states that generally have poor radiative recombination efficiency in the absence of heavy-metal atoms. There have been a few reports on plasmon-enhanced phosphorescence. ${ }^{4}$ One aspect common to these studies is that the effect was investigated in materials that are strongly spin-orbit (SO) coupled and thus

${ }^{a}$ Department of Physics and Astronomy, University of Utah,

Salt Lake City, UT 84112, USA

${ }^{b}$ Department of Chemical Sciences, Indian Institute of Science Education and Research Kolkata, Mohanpur 741252, India. E-mail:dchaudhuri@iiserkol.ac.in

${ }^{c}$ Kekulé-Institut für Organische Chemie und Biochemie der Universität Bonn, Gerhard-Domagk-Str. 1, 53121 Bonn, Germany

${ }^{d}$ Institut für Experimentelle und Angewandte Physik,

Universität Regensburg, 93053 Regensburg, Germany

$\dagger$ Electronic supplementary information (ESI) available. See DOI: $10.1039 / \mathrm{c} 2 \mathrm{cc} 31843 \mathrm{a}$ highly phosphorescent to begin with. Most hydrocarbon organic semiconductors are weakly phosphorescent. OLED electrodes provide a natural environment for surface enhancement. If surface enhancement were to apply to transitions involving pure triplet and singlet states, in the absence of SO-induced spin mixing, the mechanism could open a new intrinsic radiative channel in an OLED, changing the way triplet harvesting is achieved and removing present limitations on triplet emitters posed by organometallic chemistry. Unfortunately, as we demonstrate here, this approach will not succeed. Phosphorescence can only be enhanced by plasmonics when intersystem crossing (ISC) is already strong. Surprisingly, phosphorescence due to transitions between pure triplet and singlet states is not enhanced to any measurable extent by plasmonic effects, even though a strong increase in fluorescence is observed in dual singlet-triplet-emitting compounds.

To assess the possibility of surface enhancement of phosphorescence, we need an independent observable to confirm the presence of an enhancement effect. This observable is given by the dipole-allowed singlet transition in the dual-emitting compounds shown in Fig. 1a. We chose four materials with variable triplet yield (controlled by ISC) and singlet-triplet gap. The steady-state photoluminescence (PL) (at $300 \mathrm{~K}$ ) and delayed luminescence $(25 \mathrm{~K})$ of the compounds dispersed at low concentration in polystyrene films are shown in Fig. 1b-e. The phosphorescence yield varies strongly across the sample series. The PL spectrum of $\mathbf{1}$, a Pt-porphyrin derivative, is dominated by the $637 \mathrm{~nm}$ phosphorescence (Fig. 1b). Strong SO coupling mediated by the $\mathrm{Pt}$ centre ensures efficient and near-complete ISC to the triplet manifold. Consequently, $\mathbf{1}$ exhibits very weak fluorescence $(531 \mathrm{~nm})$ that can only be detected by using a colour filter (Schott BG39) to attenuate the phosphorescence signal. ${ }^{5} \mathbf{2}$ is a phenazine derivative that shows strong fluorescence and weaker but detectable steadystate phosphorescence at $300 \mathrm{~K}$ that is quite unusual for an organic compound devoid of any heavy atom. The phosphorescence is shifted to the red by the exchange gap $\Delta E_{\mathrm{ST}}=$ $0.6 \mathrm{eV}$. We recently reported a series of triphenylene copolymers with tuneable singlet-triplet energy difference $\Delta E_{\mathrm{ST}}{ }^{6}{ }^{6}$ To investigate whether plasmonic field-mediated singlet-triplet crossover is sensitive to $\Delta E_{\mathrm{ST}}$, we extended our study to compounds 3 

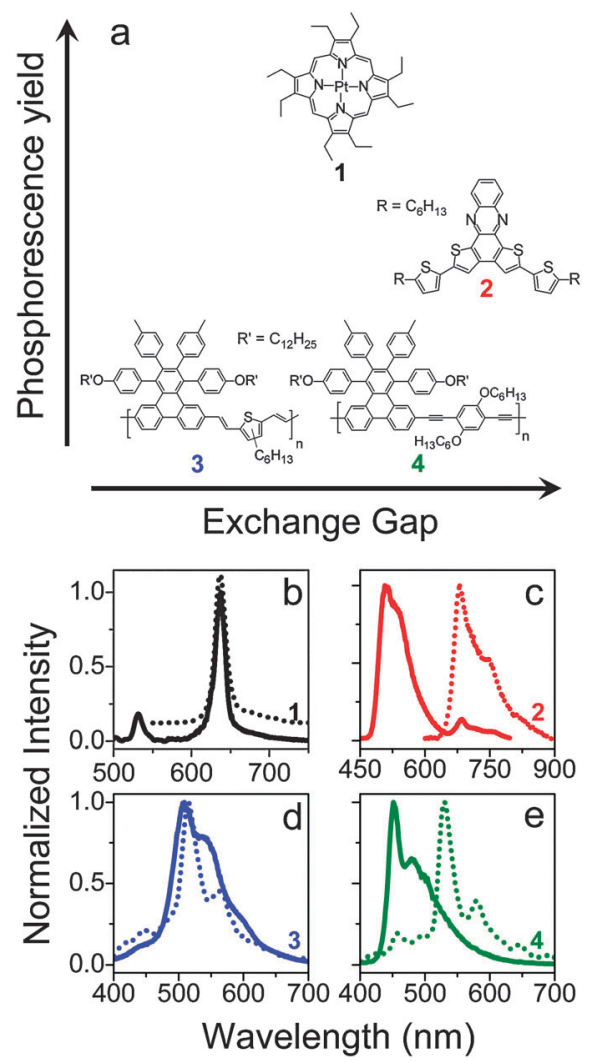

Fig. 1 Variation in phosphorescence yield of small molecules and polymers, dispersed in polystyrene films spin-coated on quartz. (a) The phosphorescence yield and exchange gap vary across the sample series. (b-e) The steady-state PL at $300 \mathrm{~K}$ (solid line) reveals the relative contribution of fluorescence and phosphorescence in each case. Delayed phosphorescence at $25 \mathrm{~K}$ (dotted line) is shown for comparison. The steady-state PL of $\mathbf{1}$ is measured through a short-pass filter to attenuate phosphorescence, the delayed phosphorescence is offset for clarity.

$\left(\Delta E_{\mathrm{ST}}=0.017 \mathrm{eV}\right)$ and $4\left(\Delta E_{\mathrm{ST}}=0.4 \mathrm{eV}\right)$. Both show weak phosphorescence that can only be detected using gated luminescence techniques at low temperatures (panels $\mathrm{d}$ and e). $\ddagger$

Silver nanoparticle films have been particularly useful as plasmonic structures in a multitude of surface-enhanced spectroscopies. ${ }^{1 a, 4 a}$ The fractal nature of these films ensures that the optical fields are concentrated into localized hotspots. Differently-sized silver nanoparticles lead to a broad plasmon resonance $^{7}$ that makes it ideal for our study, as significant overlap of the plasmon resonance with both singlet and triplet spectra of the entire sample series exists. Being a near-field effect, strongest plasmonic enhancement of the emission is expected for a chromophore lying in close proximity to the metal. This proximity can, however, introduce significant quenching through energy transfer. ${ }^{8}$ An optimal condition for emission enhancement therefore requires the chromophores to be placed at a distance from the metal surface. We achieved this by dispersing the compounds in polystyrene and coating a thin composite film on rough silver substrates.

In Fig. 2, we present the effect of plasmonic field enhancement on the decay rates of fluorescence and phosphorescence measured at the respective emission maxima. Silver nanoparticle
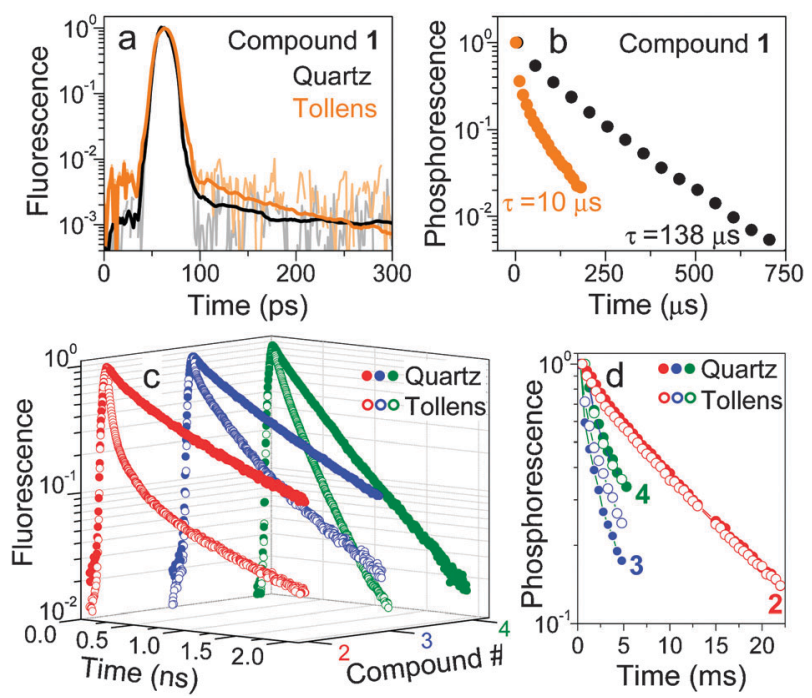

Fig. 2 Plasmonic rate enhancement of fluorescence and phosphorescence at $25 \mathrm{~K}$. The decays were measured at the respective emission maxima. (a) The fluorescence decay of $\mathbf{1}$ on quartz (black line) as well as silver (orange line) is resolution limited. (b) The weakly-forbidden phosphorescence of $\mathbf{1}$ on quartz (black circles) decays monoexponentially; the rate increases $\sim 14$-fold on silver (orange circles). (c) Fluorescence of compounds $\mathbf{2}$ (red), $\mathbf{3}$ (blue) and $\mathbf{4}$ (green) exhibits significant rate enhancements, whereas dipole-forbidden phosphorescence (d) remains unaffected on going from quartz (closed circles) to rough silver Tollens film substrates (open circles).

films were prepared following the Tollens silver mirror reaction. ${ }^{9}$ These films are known to give rise to dramatic enhancements of light-matter interaction, and have been used in conclusive demonstrations of single-molecule Raman scattering. ${ }^{9}$ The effect of heavy-atom $(\mathrm{Pt})$ mediated SO coupling discussed above is apparent in the case of $\mathbf{1}$ (panel a): the photoexcited singlet state undergoes very fast ISC to the lowest-lying triplet, the rate of which is significantly faster than that of spontaneous fluorescence. As a result, the fluorescence of $\mathbf{1}$ decays on time scales shorter than the resolution of our measurement ( $\sim 2 \mathrm{ps}$ ). Such an ultrafast decay makes it difficult to study any effect of plasmonic rate enhancement. It is important to note that interpretation of absolute emission intensities (and hence quantum yields) in terms of enhancement (or quenching) can be misleading, because the two substrates (quartz and the Tollens mirror) have very different surface roughness and wettability. Thus, the amount of sample deposited is variable, a reasonable estimate of which cannot be obtained from a routine thickness measurement. We instead compared the fluorescence to phosphorescence intensity ratio of $\mathbf{1}$ on the two surfaces at short time scales (within $25 \mathrm{ps} \S$ of excitation). In this time window, both fluorescence and phosphorescence are observed since the repetition rate of the laser $(80 \mathrm{MHz})$ is much higher than the decay rate of the triplets. Phosphorescence hence appears as a constant background to the dynamic fluorescence. A threefold increase in the fluorescence to phosphorescence ratio on the Tollens film (not shown) suggests preferential enhancement of fluorescence over phosphorescence. Such contrasting behaviour of singlet and triplet decay rates has previously been reported, ${ }^{4 f}$ albeit to a much weaker extent in an organic dye. To account for the 
effect of plasmonic field enhancement on phosphorescence alone, we compared the triplet decay rates of $\mathbf{1}$ on the two surfaces on longer time scales (panel b). The phosphorescence decay on quartz is monoexponential with a lifetime of $\sim 140 \mu \mathrm{s}$. On a Tollens film, the decay is multiexponential and the average lifetime decreases by over an order of magnitude ( $\sim 10 \mu \mathrm{s})$. The multiexponential nature of the decay suggests a statistical distribution of metal (hotspot)-chromophore separations across the excitation volume, which is to be expected given the variable roughness of the Tollens film.

Both fluorescence and phosphorescence of 1 have appreciable oscillator strength and are both enhanced on Tollens films. The situation is, however, different for the remaining compounds. We present the fluorescence intensity decay of 2, 3 and 4 in Fig. 2c. In all cases, the decay is much faster on Tollens films than on quartz. In the absence of any observed quenching, we attribute the change primarily to surface enhancement of the radiative rate. The absolute magnitude of enhancement depends intricately on the metal-chromophore interaction, a parameter that cannot be entirely reproduced by fixing the sample preparation conditions. This explains why the degree of enhancement is not the same for all samples. The fact that hotspot-chromophore separations vary across a sample is reflected in the decay curve of compound $\mathbf{2}$ on silver. One can clearly identify a very fast component corresponding to molecules experiencing varying extent of plasmonic field enhancement, and a slower component representing the unperturbed molecules with a rate similar to that on quartz. Thus, the dipoleallowed fluorescence quite expectedly shows significant enhancement of the fluorescence rate.

Phosphorescence decays of the same samples on quartz are more than three orders of magnitude slower than for $\mathbf{1}$. Although 2 exhibits a much higher phosphorescence yield than $\mathbf{3}$ and $\mathbf{4}$, which is most likely due to increased excitedstate ISC induced by the nitrogen lone-electron pair on the phenazine derivative, all three compounds show similar phosphorescence lifetimes $(\sim 3-10 \mathrm{~ms})$. Due to the lack of a heavy atom, SO coupling in these compounds is very weak and the corresponding transitions have low oscillator strength. On silver, none of the three compounds shows any appreciable change in a phosphorescence decay rate, in contrast to the case of strongly SO-coupled $\mathbf{1}$. The same samples which experience strong rate enhancement for a dipole-allowed transition (fluorescence) exhibit no noticeable effect for the dipoleforbidden one (phosphorescence). Since the measured phosphorescence decay of weakly $\mathrm{S}-\mathrm{O}$ coupled systems is dominated by non-radiative processes, any small change in the radiative rate will not affect the total decay rate. In the limit of weak SO coupling, a reduced exchange gap $\left(\Delta E_{\mathrm{ST}}\right)$ may assist spin mixing. However, our results for compounds $\mathbf{3}$ and $\mathbf{4}$ show conclusively that a plasmonic field cannot facilitate ISC for even low $\Delta E_{\mathrm{ST}}$ samples (i.e. sample 3, where singlet and triplet are effectively degenerate).

We conclude that in order to achieve plasmon-enhanced phosphorescence, the transition must have appreciable oscillator strength to begin with. Even in the presence of strong plasmonenhanced electromagnetic fields, the phosphorescence yield is limited by the strength of SO coupling. No influence of the exchange energy gap is observed. The fact that spin selection rules are so robust has important implications in designing materials for phosphorescent OLEDs, as the presence of a heavy atom to boost SO coupling remains indispensable.

We are indebted to the Volkswagen Foundation for collaborative funding. JML is a Packard Foundation Fellow.

\section{Notes and references}

$\ddagger$ Despite being close in energies, singlet and triplet emission features of $\mathbf{3}$ are distinct, which helps in unambiguous assignment of the two species. The nature of the weak band at $450 \mathrm{~nm}$ (Fig. 1d) is not clear to us. $\S$ There is no detectable fluorescence from compound $\mathbf{1}$ after 25 ps.

1 (a) V. M. Shalaev, Handbook of Nanostructured Materials and Nanotechnology, Academic Press, 1999, vol. 4; (b) J. R. Lacowicz, C. D. Geddes, I. Gryczynski, J. Malicka, Z. Gryczynski, K. Aslan, J. Lukomska, E. Matveeva, J. Zhang, R. Badugu and J. Huang, J. Fluoresc., 2004, 14, 425; (c) F. Immanuel and G. Samuel, J. Phys. D: Appl. Phys., 2008, 41, 013001; (d) M. I. Stockman, Opt. Express, 2011, 19, 22029.

2 M. Moskovits, Rev. Mod. Phys., 1985, 57, 783.

3 M. Moskovits and D. P. DiLella, J. Chem. Phys., 1982, 77, 1655.

4 (a) S. Pan and L. J. Rothberg, J. Am. Chem. Soc., 2005, 127, 6087; (b) J. C. Ostrowski, A. Mikhailovsky, D. A. Bussian, M. Summers, S. K. Buratto and G. C. Bazan, Adv. Funct. Mater., 2006, 16, 1221; (c) M. J. R. Previte, K. Aslan, Y. Zhang and C. D. Geddes, J. Phys. Chem. C, 2007, 111, 6051; (d) C. J. Yates, I. D. W. Samuel, P. L. Burn, S. Wedge and W. L. Barnes, Appl. Phys. Lett., 2006, 88, 161105; (e) T. Soller, M. Ringler, M. Wunderlich, T. A. Klar, J. Feldmann, H. P. Josel, Y. Markert, A. Nichtl and K. Kurzinger, Nano Lett., 2007, 7, 1941; (f) F. D. Stefani, K. Vasilev, N. Bocchio, F. Gaul, A. Pomozzi and M. Kreiter, New J. Phys., 2007, 9, 21.

5 J. M. Lupton, Appl. Phys. Lett., 2002, 81, 2478.

6 D. Chaudhuri, H. Wettach, K. J. vanSchooten, S. Liu, E. Sigmund, S. Höger and J. M. Lupton, Angew. Chem., Int. Ed., 2010, 49, 7714.

7 Y. Saito, J. J. Wang, D. N. Batchelder and D. A. Smith, Langmuir, 2003, 19, 6857.

8 E. Dulkeith, A. C. Morteani, T. Niedereichholz, T. A. Klar, J. Feldmann, S. A. Levi, F. C. J. M. van Veggel, D. N. Reinhoudt, M. Moeller and D. I. Gittins, Phys. Rev. Lett., 2002, 89, 203002.

9 M. J. Walter, J. M. Lupton, K. Becker, J. Feldmann, G. Gaefke and S. Höger, Phys. Rev. Lett., 2007, 98, 137401. 